\title{
Domain-elongation NMR spectroscopy yields new insights into RNA dynamics and adaptive recognition
}

\author{
QI ZHANG ${ }^{1}$ and HASHIM M. AL-HASHIMI ${ }^{2}$ \\ ${ }^{1}$ Department of Chemistry and Biochemistry, University of California at Los Angeles, Los Angeles, California, 90095, USA \\ ${ }^{2}$ Department of Chemistry and Biophysics, University of Michigan, Ann Arbor, Michigan, 48109, USA
}

\begin{abstract}
By simplifying the interpretation of nuclear magnetic resonance spin relaxation and residual dipolar couplings data, recent developments involving the elongation of RNA helices are providing new atomic insights into the dynamical properties that allow RNA structures to change functionally and adaptively. Domain elongation, in concert with spin relaxation measurements, has allowed the detailed characterization of a hierarchical network of local and collective motional modes occurring at nanosecond timescale that mirror the structural rearrangements that take place following adaptive recognition. The combination of domain elongation with residual dipolar coupling measurements has allowed the experimental threedimensional visualization of very large amplitude rigid-body helix motions in HIV-1 transactivation response element (TAR) that trace out a highly choreographed trajectory in which the helices twist and bend in a correlated manner. The dynamic trajectory allows unbound TAR to sample many of its ligand bound conformations, indicating that adaptive recognition occurs by "conformational selection" rather than "induced fit." These studies suggest that intrinsic flexibility plays essential roles directing RNA conformational changes along specific pathways.
\end{abstract}

Keywords: adaptive recognition; induced fit; tertiary capture; functional dynamics; NMR

\section{INTRODUCTION}

Ever since it was shown by X-ray crystallography that tRNA ${ }^{\text {Gln }}$ undergoes changes in the relative alignment of its helical stems upon binding to Escherichia coli glutaminyltRNA synthetase (Rould et al. 1989), it has become abundantly clear that RNA structures ubiquitously undergo adaptive conformational changes on binding to cognate targets, including ligands, small molecule therapeutics, metabolites, proteins, and other RNAs (Hermann and Patel 2000; Williamson 2000; Leulliot and Varani 2001; AlHashimi and Walter 2008). These changes not only optimize intermolecular interactions, but they also serve to sense, process, and transmit chemical information. For example, structural changes triggered by ligand binding form the basis by which many RNA riboswitches both sense and respond to environmental factors in regulating gene expression (Mandal and Breaker 2004; Montange and Batey 2008; Serganov and Patel 2008). Multistep RNA structural

Reprint requests to: Hashim M. Al-Hashimi, Department of Chemistry and Biophysics, University of Michigan, Ann Arbor, MI 48109, USA; e-mail: hashimi@umich.edu; fax: (734)647-4865.

Article published online ahead of print. Article and publication date are at http://www.rnajournal.org/cgi/doi/10.1261/rna.1806909. transitions can help order the sequence of transactions underlying assembly of ribonucleoproteins and catalysis (Liu et al. 2007; Stone et al. 2007; Williamson 2008; Woodson 2008). Structural transitions can also be used to inhibit the activity of RNA. For example, the antibiotic activity of aminoglycosides is largely derived from their ability to flip out two internal loop adenines in the ribosomal A-site (Fourmy et al. 1996), which serves to disrupt decoding and the fidelity of translation (Vicens and Westhof 2003; Hermann 2006).

Over the past two decades, there has been great interest in understanding the mechanism by which RNA structures undergo adaptive recognition (Hermann and Patel 2000; Williamson 2000; Leulliot and Varani 2001; Al-Hashimi 2005). On the one hand, these studies are motivated by a desire to understand how RNA structures change in response to specific cellular signals while avoiding promiscuous transitions from taking place. On the other hand, having the ability to predict how RNA structures change adaptively upon binding to small molecules is key for rationally designing RNA-binding therapeutics. Two nonmutually exclusive mechanisms have been used to explain RNA adaptive recognition (Fig. 1; Frankel and Smith 1998; Williamson 2000; Leulliot and Varani 2001). The induced-fit 


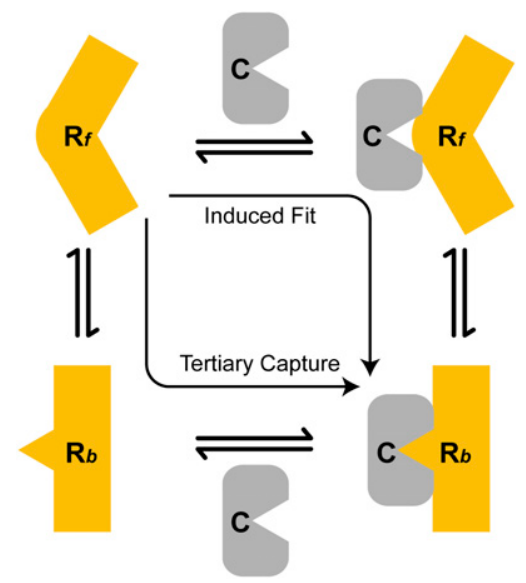

FIGURE 1. Schematic representation of induced-fit and conformational selection/tertiary capture mechanisms for RNA (R) adaptive recognition upon binding to cofactors $(\mathrm{C})$.

model, which was introduced by Koshland in the 1950s to explain the much lower rates of protein enzyme catalysis observed with substrate analogs (Koshland 1958), was used to explain most of early observations of RNA adaptive recognition. Here, it is assumed that the RNA bound conformation is not significantly populated in the absence of the binding partner, either because it is energetically unfavorable or because it is separated from the ground state by very large kinetic barriers, and that binding partners are required to effect the RNA conformational change (Fig. 1). However, there were also studies that provided experimental evidence that binding partners do not induce the RNA conformational change but rather capture pre-existing RNA conformations (Fig. 1). An early example of RNA conformational selection or tertiary capture, which was first introduced by Foote and Milstein (1994) to explain kinetic data on a set of antigen-antibody interactions, was the observation that complexes formed by complementary RNA stem-loops are stabilized upon binding to the ColE1 Rom protein (Eguchi and Tomizawa 1990). Weeks and Cech $(1995,1996)$ also invoked tertiary capture to explain adaptive recognition during the assembly of CBP2-group I intron ribonucleoprotein complex, in which proteins bind rapidly and capture transiently formed RNA tertiary conformations along the assembly pathway.

Whether by induced fit, conformational selection, or both, a complete understanding of how RNA structures change adaptively requires the characterization of RNA's intrinsic structural flexibility at the atomic level (Leulliot and Varani 2001; Al-Hashimi 2005; Al-Hashimi and Walter 2008; Hall 2008). Regulatory RNAs must encode-at some level-flexibility that allows their structures to change specifically along functionally productive pathways. This flexibility is imprinted in the thermal fluctuations of RNA structure over picosecond-to-second timescales. The past decade has witnessed great developments in biophysical techniques for characterizing internal motions in RNA. These include ultrafast spectroscopy (Xia 2008), computational molecular dynamics simulation (Chen 2008; Hall 2008; Mackerell and Nilsson 2008), single molecule techniques ( $\mathrm{Li}$ et al. 2008), time-resolved hydroxyl radical footprinting techniques with millisecond resolution (Sclavi et al. 1998; Shcherbakova et al. 2006), and both solution and solid-state nuclear magnetic resonance (NMR) (Furtig et al. 2007; Getz et al. 2007a; Shajani and Varani 2007). In this mini-review, we focus on recent developments in solution NMR spectroscopy that involve elongating RNA targets, are making possible the quantitative site-specific characterization of dynamics over picosecond-to-millisecond timescales, and are helping unveil the rich complexity of RNA flexibility at atomic resolution and its role in conformational adaptation.

\section{DOMAIN ELONGATION: DECOUPLING INTERNAL AND OVERALL MOTIONS IN NMR STUDIES OF RNA DYNAMICS}

Consider the task of reconstructing thermal motions of a complex biomolecule at atomic resolution over biologically relevant timescales based on experimental data; compared with solving the structure of a single snapshot, one requires a factor of $\sim 10^{12}$ more data to solve a onesecond dynamic trajectory with picosecond resolution. Measuring such a mind-boggling amount of data is currently impossible by any experimental technique. By allowing measurements of not one but many complementary interactions on a per site basis, NMR spectroscopy offers a unique opportunity to obtain a glimpse into the time-space complexity of RNA dynamics. NMR spectroscopy has greatly contributed to our understanding of protein dynamics and its role in folding and function (Bruschweiler 2003; Kern and Zuiderweg 2003; Palmer 2004; Mittermaier and Kay 2006; Henzler-Wildman and Kern 2007). However, most NMR methods for dynamics characterization have been developed in application to proteins, and they often cannot be directly applied to RNA, which has distinct spectroscopic and hydrodynamical properties (Al-Hashimi 2005; Latham et al. 2005).

NMR data, such as spin relaxation rates, which can be used to probe motions occurring at picosecond-to-nanosecond timescales (Lipari and Szabo 1982), and residual dipolar couplings (RDCs) (Tolman et al. 1995; Tjandra and Bax 1997), which can be used to probe motions over submillisecond timescales (Tolman et al. 1997; Getz et al. 2007a), fundamentally probe the reorientation of anisotropic spin interactions, which are centered on a given nucleus, relative to the external magnetic field. For example, spin relaxation rates and RDCs report on fluctuations that change the orientation of a given $\mathrm{C}-\mathrm{H}$ bond relative to the external magnetic field. In solution NMR, reorientation of the $\mathrm{C}-\mathrm{H}$ bond is dominated by overall rotational 
diffusion of the biomolecule. However, internal motions can also contribute-albeit by a smaller amount-to the reorientation of $\mathrm{C}-\mathrm{H}$ bond and thereby influence spin relaxation rates and RDCs. The internal and overall motional contributions to NMR measurements can be disentangled provided one can assume that they are not correlated to one another. While this so-called "decoupling approximation" (Lipari and Szabo 1982) has been shown to hold to a high degree of validity for many globular proteins (Peti et al. 2002; Tolman 2002), studies over the past decade have shown this not to be the case for highly flexible RNAs (Zhang et al. 2003, 2006; Showalter and Hall 2005; Musselman et al. 2007). In RNA, collective movements of A-form helices linked by flexible junctions can occur at timescales approaching overall rotational diffusion and cause large deformations in the overall hydrodynamic molecular shape and therefore overall reorientation (Fig. 2A). The ensuing motional couplings make it difficult, if not impossible, to separate the internal and overall motional contributions and thus to extract the desired quantitative information regarding the internal dynamics.

In our laboratory, we have developed a domain-elongation strategy for decoupling internal and overall motions in RNA (Zhang et al. 2006). In this approach, a terminal helix is elongated using a stretch of Watson-Crick base pairs, designed to adopt an A-form helix structure (Fig. 2A). This renders the overall shape of the elongated RNA, and therefore overall reorientation, far less sensitive to internal motions occurring in other parts of the molecule. By slowing down the overall rate of molecular tumbling, the elongation serves to broaden the timescale sensitivity of spin relaxation measurements, which is limited to internal motions occurring at timescales faster than overall tumbling. The elongation also predefines overall motions to be axially symmetric with principal direction oriented along the elongated axis. This further simplifies the characterization of the overall motions and the quantitative interpretation of NMR data in terms of internal motions. A key feature of the domain-elongation strategy is that it allows computation of measured NMR spin relaxation and RDC parameters, for any given dynamic trajectory, regardless of its complexity. This proves to be particularly useful in comparing NMR data with molecular dynamics simulations (Musselman et al. 2007; Frank et al. 2009).

An isotopic labeling strategy was devised in order to avoid unmanageable NMR spectral overcrowding arising due to elongation residues (Zhang et al. 2006). The strategy involves the preparation of two constructs in which stretches of unlabeled A-U (E-AU-RNA) and G-C (E-GC-RNA) base pairs are employed for elongation using otherwise uniformly
A

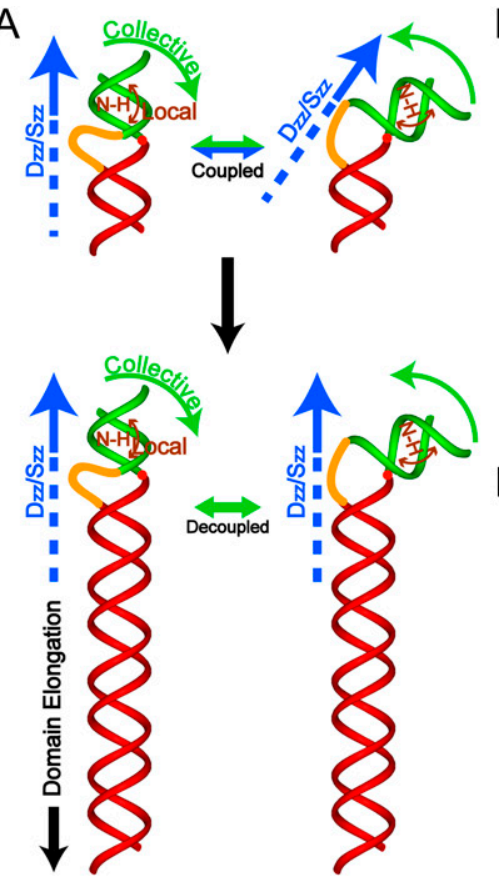

B

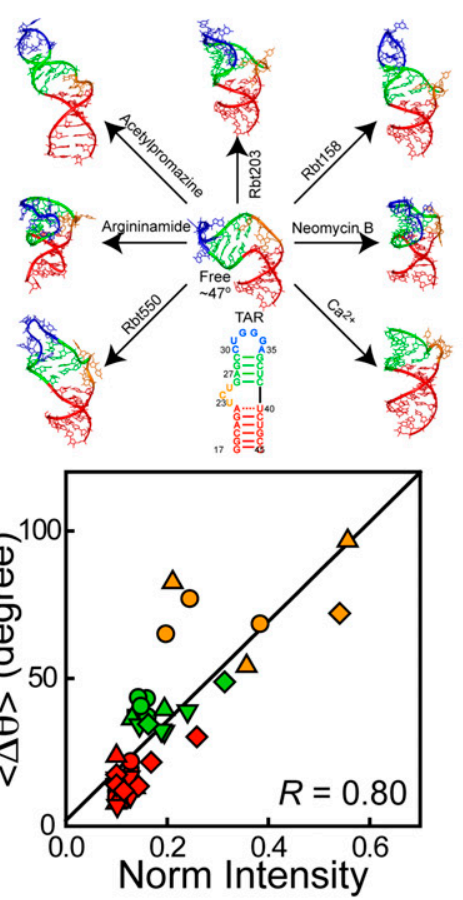

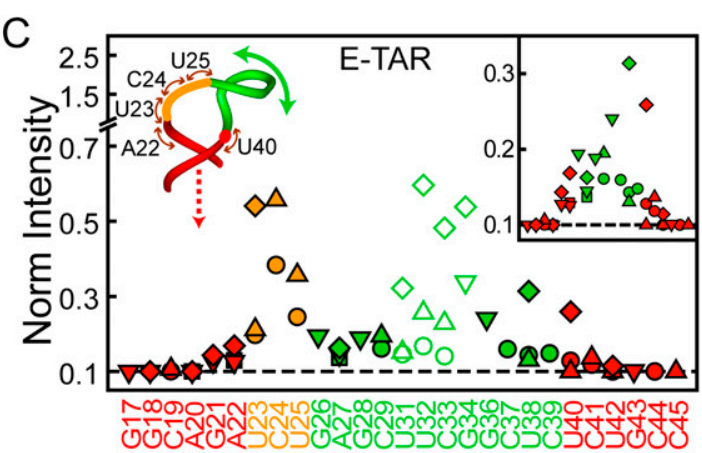

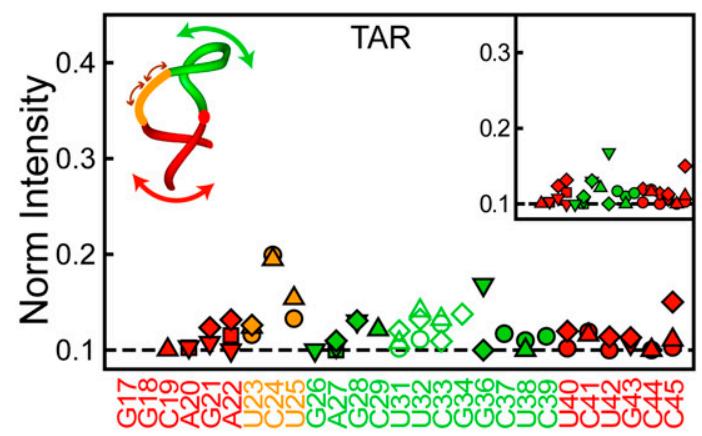

FIGURE 2. Domain elongation and NMR spin relaxation reveal nanosecond motions in HIV-1 TAR RNA (Zhang et al. 2006). (A) Collective motions of helices can lead to coupled changes in the overall rotational diffusion $\left(D_{z z}\right)$ and/or partial alignment $\left(S_{z z}\right)$ of the RNA, which can be decoupled by domain elongation. (B) TAR conformational adaptation upon binding to different ligands and small molecules. (C) Resolving a hierarchical network of local and collective motional modes in TAR RNA. Shown are normalized sugar and base C-H resonance intensities (peak heights) measured from nonconstant time two-dimensional ${ }^{1} \mathrm{H}-{ }^{13} \mathrm{C}$ HSQC spectra E-TAR and TAR. $(D)$ The intrinsic dynamics underlies the conformational adaptation. Correlation plot between the mean-angular-difference $(\langle\Delta \theta\rangle)$ in the orientation of sugar and base $\mathrm{C}-\mathrm{H}$ bond vectors across eight different HIV-1 TAR structures and the corresponding free E-TAR normalized resonance intensities. 
${ }^{13} \mathrm{C} /{ }^{15} \mathrm{~N}$-labeled G-C and A-U nucleotides, respectively. The two constructs thus allow acquisition of NMR data over the entire RNA target while keeping elongation residue NMRs "invisible." Of key importance is that the elongation does not affect the structural and functional integrity of the studied RNA. Thus far, comparison of nonelongated and elongated NMR spectra for a variety of RNA contexts under free and ligand or metal bound conditions suggest that elongation does not affect the structural, dynamical, and functional integrity of the targeted RNAs (Zhang et al. 2006; Getz et al. 2007b; Shajani et al. 2007; Sun et al. 2007; Bardaro et al. 2009).

\section{DOMAIN-ELONGATION AND SPIN RELAXATION MEASUREMENTS EXPOSE NANOSECOND INTERNAL MOTIONS IN RNA}

Domain elongation was first used to study the dynamical properties of the transactivation response element (TAR) (Muesing et al. 1987), which is a stem-loop located at the 5 ' -end of the HIV-1 RNA genome that plays essential roles in various steps of the viral replication cycle. Early studies by Williamson, Puglisi, and Varani established that TAR is a highly adaptable molecule that undergoes large structural rearrangements upon binding to peptides and ligands derived from the TAR cognate target the transactivator protein Tat (Puglisi et al. 1992; Aboul-ela et al. 1995, 1996). Subsequently, structures were reported for TAR bound to $\mathrm{Mg}^{2+}$ ions (Ippolito and Steitz 1998) and five different small molecule inhibitors of the TAR-Tat interaction (Faber et al. 2000; Du et al. 2002; Davis et al. 2004; Murchie et al. 2004). These TAR structures superimpose with an allatom root-mean-square deviation of $4.7 \AA$ and differ both in the global orientation of helical domains (interhelical angle ranging between $\sim 5^{\circ}$ and $\sim 47^{\circ}$ ) and local structure of the binding pocket comprising bulge and neighboring residues (Fig. 2B). A key question that has important implications on the rational design of TAR binding antiHIV therapeutics is whether various ligands induce distinct TAR conformations via induced-fit or bind pre-existing conformations from a dynamical ensemble by conformational selection.

Domain-elongation NMR spectroscopy was used to obtain insights into the dynamical properties of HIV-1 TAR (Zhang et al. 2006). Despite the agreement between NMR spectra of elongated and nonelongated TAR, the spin relaxation properties of elongated TAR (E-TAR) exposed complex motional modes that evade detection in the nonelongated construct due to motional couplings. This complex motional manifold appeared as large variations in the resonance intensities of E-TAR, which, ignoring chemical exchange, report the net dynamics of a given site relative to the applied magnetic field. Unusually high intensities were observed for bulge and neighboring residues, indicating that the interhelix interface is highly flexible (Fig. 2C). The consistently higher intensities observed for the short helix compared with the elongated helix provided evidence that the helices move collectively across the flexible interface (Fig. 2C). These motions were not resolved in spectra of nonelongated TAR due to motional couplings. A similar pattern of intensities was resolved in a different HIV-1 element, SL1, indicating that these nanosecond local and collective motional modes are a general feature of RNA architecture. Model-free analysis of ${ }^{15} \mathrm{~N}$ relaxation measured in E-TAR and E-SL1 confirmed the presence of collective helix motions with amplitudes corresponding to spin relaxation order parameters of $S_{s}{ }^{2}=$ $0.68-0.83$ (the value of $S^{2}$ ranges between 0 , for maximum motions, and 1 , for rigidity) that move with time constants $\left(\tau_{s}=1.4-1.9 \mathrm{nsec}\right)$ approaching the hydrodynamically predicted time constants for their overall rotational diffusion.

How do the motions uncovered in TAR relate to its ability to adopt different conformations and thereby bind to different small molecules and ligands? Interestingly, a correlation was observed on a site-by-site basis between the observed dynamics at a given site, as measured qualitatively based on resonance intensities, and the amplitude of the rearrangements the site undergoes upon binding to different ligands (Fig. 2D). These results provided evidence that TAR codes for intrinsic dynamics that allows its conformation to change functionally and adaptively.

\section{VISUALIZING SUBMILLISECOND HELIX MOTIONS IN HIV-1 TAR USING DOMAIN ELONGATION AND RDCS}

The spin relaxation data suffer from two limitations. First, sensitivity is limited to motions occurring at timescales faster than overall tumbling, i.e., tens of nanoseconds. Second, while insights can be obtained regarding the amplitudes of motions, it is generally difficult to reconstruct the spatial characteristics of the underlying trajectory, which are needed, for example, to establish whether unbound TAR can dynamically sample its ligand bound conformations. The measurement of RDCs can fill this gap (Tjandra and Bax 1997; Prestegard et al. 2000). They are sensitive to motions occurring at submillisecond timescales and, when internal motions are decoupled from overall alignment, as afforded by domain elongation, they can readily be interpreted in terms of a dynamical distribution of bond vector orientations relative to an alignment or order tensor describing overall alignment. RDCs are particularly powerful at characterizing rigid-body motions, such as collective motions of helices in RNA (Al-Hashimi et al. 2002; Musselman et al. 2006). In this case, collections of RDCs measured throughout the helix can be analyzed in concert, thus sharpening the spatial resolution with which the underlying dynamic trajectory can be characterized (Tolman et al. 1997). 
Domain elongation greatly simplifies the dynamical interpretation of RDCs. In particular, the elongations render RDCs dependent on the angle $(\Omega)$ between bond vectors and the internal elongated axis and not a detached external magnetic field (Zhang et al. 2007). For a given elongated RNA, RDCs can be used to probe interhelical bending $(\beta)$ and twisting $(\alpha)$ motions of the short helix; however, they are insensitive to twisting motions $(\gamma)$ around the axially symmetric elongated helix (Fig. 3A). To resurrect this vanishing sensitivity, and thereby measure collective helix motion complete three-dimensional (3D) rotational sensitivity, a second elongated construct is prepared in which the short helix is now elongated. In this construct, the RDCs can probe variations in the angles $\beta$ and $\gamma$, but not $\alpha$ (Fig. $3 \mathrm{~A})$. Thus, assuming that the two different elongations do not perturb the motions, the measurement of RDCs in the two elongated samples makes it possible to characterize interhelix motions with complete 3D rotational sensitivity.

Domain elongation and inverse domain elongation coupled with RDC measurements were used to visualize interhelical motions in HIV -1 TAR. An order tensor analysis of RDCs revealed super large amplitude helix motions $\left(\vartheta_{\text {int }} \sim 0.45\right.$ and 0.47 , for EI-TAR and EII-TAR, respectively) that significantly exceed the amplitudes observed previously by spin relaxation $\left(\sqrt{S_{s}^{2}}=\vartheta_{\text {int }} \sim 0.86\right)$ (Zhang et al. 2006). Given the broader timescale sensitivity of RDCs $(<0.1 \mathrm{msec})$ compared with spin relaxation $(<19$ nsec) and absence of significant exchange broadening in and around the bulge (Zhang et al. 2006; Dethoff et al. 2008), the motions most likely occur at the nanosecondmicrosecond timescale.
The RDCs measured in the two elongated TAR samples were used to construct interhelical ensembles consisting of up to three conformations (Zhang et al. 2007). No single interhelical conformation could be determined that satisfied the measured RDCs within experimental precision; likewise, no two-state ensemble could be determined that satisfied the measured RDCs even when allowing for asymmetric populations. However, three-state ensembles with equal populations could be identified that satisfied the measured RDCs close to within experimental precision. Cluster analysis of these ensembles revealed that the three conformers consistently trace out a spatially choreographed trajectory in which the two helices twist and bend in a spatially correlated manner (Fig. 3B). Thus, although the helix motions have very large amplitudes, they are not spatially random. Strikingly, the seven distinct ligand bound TAR conformations fell along various positions of this dynamic trajectory, indicating that ligands most likely capture pre-existing TAR conformations by conformational selection rather than induce new ones by "induced fit" (Fig. 3C).

\section{OTHER APPLICATIONS AND DEVELOPMENTS IN DOMAIN-ELONGATION NMR SPECTROSCOPY}

A growing number of studies have used domain-elongation and spin relaxation measurements to uncover local motions that are slower than overall tumbling (so-called supra- $\tau_{m}$ motions) in diverse RNA contexts. For example, domain elongation exposed near- $\tau_{\mathrm{m}}$ local motions in an extruded uridine bulge residue in the $\mathrm{P} 4$ helix of RNase $\mathrm{P}$
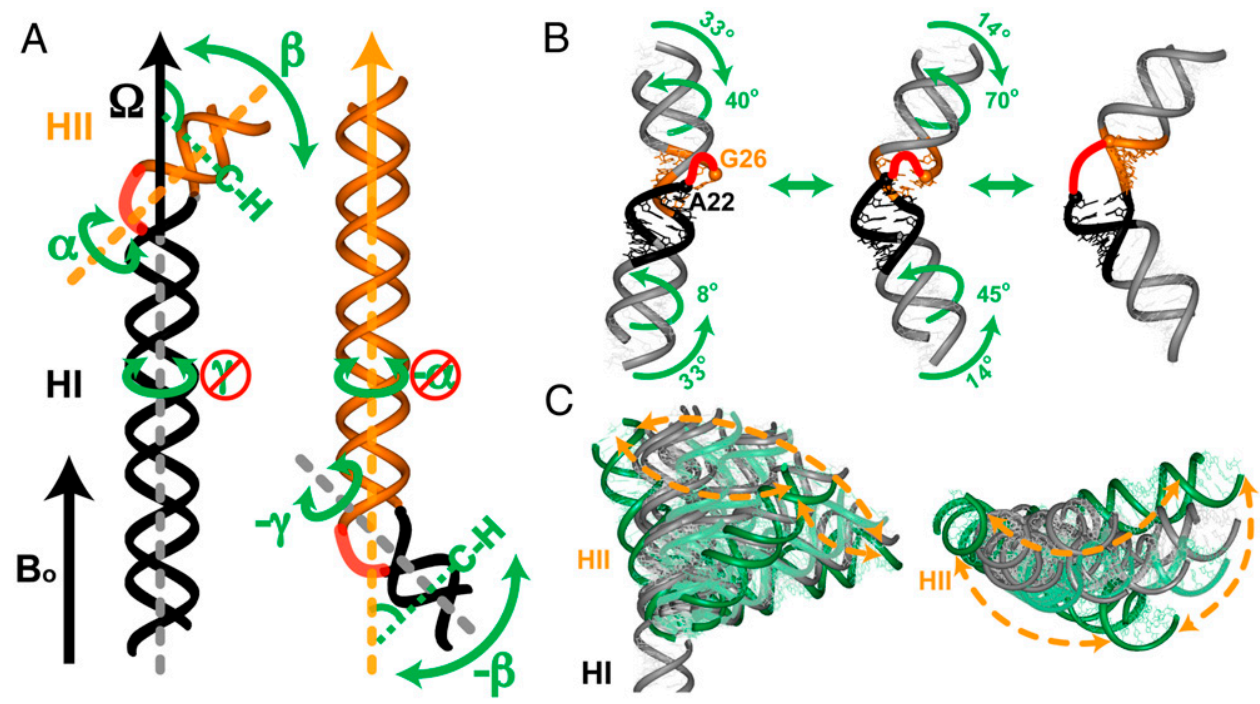

FIGURE 3. Visualizing collective helix motions in TAR RNA using domain elongation (Zhang et al. 2007). (A) Domain and inverse domain elongation for visualizing interhelical motions with 3D rotational sensitivity to twisting $(\alpha$ and $\gamma$ ) and bending $(\beta)$ motions. (B) Transitions between the three TAR ensemble conformers give rise to correlated twisting and bending dynamics. $(C)$ Comparison of the three TAR ensemble conformers (in green) and ligand bound TAR conformations (in gray). Subconformers along the linear pathway linking three ensemble conformers are shown in light green and the direction of the trajectory is shown in orange arrows. 
that completely evades detection in nonelongated P4 (Getz et al. 2007b). Likewise, it helped uncover local motions in two guanine internal loop residues in HIV-1 SL1 that are activated upon $\mathrm{Mg}^{2+}$ binding and that may play an important role in adaptively binding the nucleocapsid protein (Sun et al. 2007). Domain elongation also revealed an unexpected increase in local flexibility of RNA residues located within a binding site induced by binding of U1A protein (Shajani et al. 2007). A similar increase in mobility was observed in the TAR bulge on binding argininamide and other ligands (Bardaro et al. 2009). Domain elongation has also helped resolve near- $\tau_{\mathrm{m}}$ local motions within the wild-type TAR apical loop that are superimposed onto slower microsecond-millisecond motions (Dethoff et al. 2008). Together, these studies have also helped establish the generality of collective helix motions across a variety of junctions. Results so far suggest that the amplitude of interhelical motions increase with the length of the interhelical linker. New methods have also been introduced that permit quantitative measurement and analysis of sugar and base ${ }^{13} \mathrm{C}$ spin relaxation data in large elongated RNAs, thus significantly increasing the probes available to quantitatively characterize motions, particularly for functionally important internal loops and bulges for which ${ }^{15} \mathrm{~N}$ relaxation measurements are generally not feasible (Hansen and Al-Hashimi 2007).

There have also been developments in the application of domain elongation and RDCs. Theoretical frameworks have been introduced to extract the maximum information contained in RDCs measured from differentially elongated RNA samples, which also make it possible to establish the correlated nature of motions involving more than two helices (Fisher et al. 2008). An approach has also developed to approximately reconstruct spatially complex continuous motions of chiral domains, such as helix motions in RNA, using multialignment RDCs (Fisher and Al-Hashimi 2009). This study showed that it is theoretically possible to experimentally reconstruct salient features of a multisegment continuous interhelical motional trajectory obtained from a molecular dynamics (MD) simulation of TAR. More recently, a new strategy has been developed that combines RDCs measured in elongated RNA with computational MD simulations (Frank et al. 2009). This approach was successfully used to generate all-atom structural ensembles of HIV-1 and HIV-2 TAR with timescale sensitivity extending up to milliseconds. The ensemble allowed visualization of local motions in the internal bulge and neighboring residues that activate the collective interhelical dynamics and provided further support that unbound TAR can dynamically sample its ligand bound conformation. Together, these studies suggest that the synergistic combination of NMR experimental data and computational MD simulations will make it possible to meet the challenge of acquiring the parameters needed to construct atomic movies of RNA structures in action.

\section{CONCLUDING REMARKS}

By providing quantitative insights into the amplitudes, timescales, and spatial choreography of motions with timescale sensitivity extending from picoseconds up to milliseconds, domain-elongation NMR spectroscopy is greatly contributing toward our understanding of RNA dynamics and its role in function. It is now clear that motions in RNA are as complex as those observed in proteins, featuring local and collective motional modes spanning picosecond-second timescales and that trace out spatially complex trajectories. There is a need to classify the various types of motional modes so as to better understand the relationship between motions and RNA structure on the one hand, and motions and RNA function on the other. For example, it is tempting to speculate that changes in interhelical orientation that do not feature changes in secondary structure, including those observed for tRNA ${ }^{\text {Gln }}$ on binding to glutaminyl-tRNA synthetase two decades ago, universally occur by conformational selection rather than induced fit. Domain-elongation NMR spectroscopy will undoubtedly play an important role uncovering these dynamic characteristics of RNA structure at atomic resolution. When combined with other developments in timeresolved NMR spectroscopy for directly monitoring RNA structural transitions at atomic resolution (Furtig et al. 2007) and solid-state techniques for high-resolution spatiotemporal characterization of motions (Miller et al. 2006; Olsen et al. 2008), the future of NMR in studies of RNA dynamics appears very promising.

\section{ACKNOWLEDGMENTS}

This work was supported by NIH and NSF grants to H.M.A.-H. (RO1 AI066975-01 and MCB 0644278). Q.Z. is a Baltimore Family Fellow of the Life Sciences Research Foundation. We thank present and past members of the Al-Hashimi laboratory for their contributions to various aspects of domain-elongation NMR spectroscopy reviewed here. The Al-Hashimi laboratory acknowledges collaborations with the laboratories of Professor Ioan Andricioaei (UC Irvine) and Professor Carol Fierke (University of Michigan).

\section{REFERENCES}

Aboul-ela F, Karn J, Varani G. 1995. The structure of the humanimmunodeficiency-virus type-1 TAR RNA reveals principles of RNA recognition by Tat protein. J Mol Biol 253: 313-332.

Aboul-ela F, Karn J, Varani G. 1996. Structure of HIV-1 TAR RNA in the absence of ligands reveals a novel conformation of the trinucleotide bulge. Nucleic Acids Res 24: 3974-3981.

Al-Hashimi HM. 2005. Dynamics-based amplification of RNA function and its characterization by using NMR spectroscopy. ChemBioChem 6: 1506-1519.

Al-Hashimi HM, Walter NG. 2008. RNA dynamics: It is about time. Curr Opin Struct Biol 18: 321-329.

Al-Hashimi HM, Gosser Y, Gorin A, Hu W, Majumdar A, Patel DJ. 2002. Concerted motions in HIV-1 TAR RNA may allow access to bound state conformations: RNA dynamics from NMR residual dipolar couplings. J Mol Biol 315: 95-102. 
Bardaro MF Jr, Shajani Z, Patora-Komisarska K, Robinson JA, Varani G. 2009. How binding of small molecule and peptide ligands to HIV-1 TAR alters the RNA motional landscape. Nucleic Acids Res 37: 1529-1540.

Bruschweiler R. 2003. New approaches to the dynamic interpretation and prediction of NMR relaxation data from proteins. Curr Opin Struct Biol 13: 175-183.

Chen SJ. 2008. RNA folding: Conformational statistics, folding kinetics, and ion electrostatics. Annu. Rev. Biophys. 37: 197-214.

Davis B, Afshar M, Varani G, Murchie AI, Karn J, Lentzen G, Drysdale M, Bower J, Potter AJ, Starkey ID, et al. 2004. Rational design of inhibitors of HIV-1 TAR RNA through the stabilization of electrostatic 'hot spots.' J Mol Biol 336: 343-356.

Dethoff EA, Hansen AL, Musselman C, Watt ED, Andricioaei I, AlHashimi HM. 2008. Characterizing complex dynamics in the transactivation response element apical loop and motional correlations with the bulge by NMR, molecular dynamics, and mutagenesis. Biophys J 95: 3906-3915.

Du Z, Lind KE, James TL. 2002. Structure of TAR RNA complexed with a Tat-TAR interaction nanomolar inhibitor that was identified by computational screening. Chem Biol 9: 707-712.

Eguchi Y, Tomizawa J. 1990. Complex formed by complementary RNA stem-loops and its stabilization by a protein: Function of CoIE1 Rom protein. Cell 60: 199-209.

Faber C, Sticht H, Schweimer K, Rosch P. 2000. Structural rearrangements of HIV-1 Tat-responsive RNA upon binding of neomycin B. J Biol Chem 275: 20660-20666.

Fisher CK, Al-Hashimi HM. 2009. Approximate reconstruction of continuous spatially complex domain motions by multialignment NMR residual dipolar couplings. J Phys Chem B 113: 6173-6176.

Fisher CK, Zhang Q, Stelzer A, Al-Hashimi HM. 2008. Ultrahigh resolution characterization of domain motions and correlations by multialignment and multireference residual dipolar coupling NMR. J Phys Chem B 112: 16815-16822.

Foote J, Milstein C. 1994. Conformational isomerism and the diversity of antibodies. Proc Natl Acad Sci 91: 10370-10374.

Fourmy D, Recht MI, Blanchard SC, Puglisi JD. 1996. Structure of the A site of Escherichia coli 16S ribosomal RNA complexed with an aminoglycoside antibiotic. Science 274: 1367-1371.

Frank AT, Stelzer AC, Al-Hashimi HM, Andricioaei I. 2009. Constructing RNA dynamical ensembles by combining $\mathrm{MD}$ and motionally decoupled NMR RDCs: New insights into RNA dynamics and adaptive ligand recognition. Nucleic Acids Res 37: 3670-3679.

Frankel AD, Smith CA. 1998. Induced folding in RNA-protein recognition: More than a simple molecular handshake. Cell 92: 149-151.

Furtig B, Buck J, Manoharan V, Bermel W, Jaschke A, Wenter P, Pitsch S, Schwalbe H. 2007. Time-resolved NMR studies of RNA folding. Biopolymers 86: 360-383.

Getz M, Sun X, Casiano-Negroni A, Zhang Q, Al-Hashimi HM. 2007a. NMR studies of RNA dynamics and structural plasticity using NMR residual dipolar couplings. Biopolymers 86: 384-402.

Getz MM, Andrews AJ, Fierke CA, Al-Hashimi HM. 2007b. Structural plasticity and $\mathrm{Mg}^{2+}$ binding properties of RNase P P4 from combined analysis of NMR residual dipolar couplings and motionally decoupled spin relaxation. RNA 13: 251-266.

Hall KB. 2008. RNA in motion. Curr Opin Chem Biol 12: 612-618.

Hansen AL, Al-Hashimi HM. 2007. Dynamics of large elongated RNA by NMR carbon relaxation. J Am Chem Soc 129: 16072-16082.

Henzler-Wildman K, Kern D. 2007. Dynamic personalities of proteins. Nature 450: 964-972.

Hermann T. 2006. A-site model RNAs. Biochimie 88: 1021-1026.

Hermann T, Patel DJ. 2000. Adaptive recognition by nucleic acid aptamers. Science 287: 820-825.

Ippolito JA, Steitz TA. 1998. A 1.3-Å resolution crystal structure of the HIV-1 trans-activation response region RNA stem reveals a metal ion-dependent bulge conformation. Proc Natl Acad Sci 95: 98199824.
Kern D, Zuiderweg ER. 2003. The role of dynamics in allosteric regulation. Curr Opin Struct Biol 13: 748-757.

Koshland DEJ. 1958. Application of a theory of enzyme specificity to protein synthesis. Proc Natl Acad Sci 44: 98-104.

Latham MP, Brown DJ, McCallum SA, Pardi A. 2005. NMR methods for studying the structure and dynamics of RNA. ChemBioChem 6: 1492-1505.

Leulliot N, Varani G. 2001. Current topics in RNA-protein recognition: Control of specificity and biological function through induced fit and conformational capture. Biochemistry 40: 79477956.

Li PT, Vieregg J, Tinoco I Jr. 2008. How RNA unfolds and refolds. Annu Rev Biochem 77: 77-100.

Lipari G, Szabo A. 1982. Model-free approach to the interpretation of nuclear magnetic resonance relaxation in macromolecules. 1. Theory and range of validity. J Am Chem Soc 104: 4546-4559.

Liu S, Bokinsky G, Walter NG, Zhuang X. 2007. Dissecting the multistep reaction pathway of an RNA enzyme by singlemolecule kinetic 'fingerprinting.' Proc Natl Acad Sci 104: 1263412639.

Mackerell AD Jr, Nilsson L. 2008. Molecular dynamics simulations of nucleic acid-protein complexes. Curr Opin Struct Biol 18: 194-199.

Mandal M, Breaker RR. 2004. Gene regulation by riboswitches. Nat Rev Mol Cell Biol 5: 451-463.

Miller PA, Shajani Z, Meints GA, Caplow D, Goobes G, Varani G, Drobny GP. 2006. Contrasting views of the internal dynamics of the HhaI methyltransferase target DNA reported by solution and solid-state NMR spectroscopy. J Am Chem Soc 128: 15970-15971.

Mittermaier A, Kay LE. 2006. New tools provide new insights in NMR studies of protein dynamics. Science 312: 224-228.

Montange RK, Batey RT. 2008. Riboswitches: Emerging themes in RNA structure and function. Annu. Rev. Biophys. 37: 117-133.

Muesing MA, Smith DH, Capon DJ. 1987. Regulation of mRNA accumilation by a human immunodeficiency virus trans-activator protein. Cell 48: 691-701.

Murchie AI, Davis B, Isel C, Afshar M, Drysdale MJ, Bower J, Potter AJ, Starkey ID, Swarbrick TM, Mirza S, et al. 2004. Structure-based drug design targeting an inactive RNA conformation: Exploiting the flexibility of HIV-1 TAR RNA. J Mol Biol 336: 625-638.

Musselman C, Pitt SW, Gulati K, Foster LL, Andricioaei I, AlHashimi HM. 2006. Impact of static and dynamic A-form heterogeneity on the determination of RNA global structural dynamics using NMR residual dipolar couplings. J Biol NMR 36: 235-249.

Musselman C, Al-Hashimi HM, Andricioaei I. 2007. iRED analysis of TAR RNA reveals motional coupling, long-range correlations, and a dynamical hinge. Biophys J 93: 411-422.

Olsen GL, Echodu DC, Shajani Z, Bardaro MF Jr, Varani G, Drobny GP. 2008. Solid-state deuterium NMR studies reveal micros-ns motions in the HIV-1 transactivation response RNA recognition site. J Am Chem Soc 130: 2896-2897.

Palmer AG 3rd. 2004. NMR characterization of the dynamics of biomacromolecules. Chem Rev 104: 3623-3640.

Peti W, Meiler J, Bruschweiler R, Griesinger C. 2002. Model-free analysis of protein backbone motion from residual dipolar couplings. J Am Chem Soc 124: 5822-5833.

Prestegard JH, Al-Hashimi HM, Tolman JR. 2000. NMR structures of biomolecules using field oriented media and residual dipolar couplings. Q Rev Biophys 33: 371-424.

Puglisi JD, Tan R, Calnan BJ, Frankel AD, Williamson JR. 1992. Conformation of the TAR RNA-arginine complex by NMR spectroscopy. Science 257: 76-80.

Rould MA, Perona JJ, Soll D, Steitz TA. 1989. Structure of E. coli glutaminyl-tRNA synthetase complexed with tRNA ${ }^{\text {Gln }}$ and ATP at $2.8 \AA$ resolution. Science 246: $1135-1142$.

Sclavi B, Sullivan M, Chance MR, Brenowitz M, Woodson SA. 1998. RNA folding at millisecond intervals by synchrotron hydroxyl radical footprinting. Science 279: 1940-1943. 
Serganov A, Patel DJ. 2008. Toward deciphering the principles underlying an mRNA recognition code. Curr Opin Struct Biol 18: $120-129$.

Shajani Z, Varani G. 2007. NMR studies of dynamics in RNA and DNA by 13C relaxation. Biopolymers 86: 348-359.

Shajani Z, Drobny G, Varani G. 2007. Binding of U1A protein changes RNA dynamics as observed by 13C NMR relaxation studies. Biochemistry 46: 5875-5883.

Shcherbakova I, Mitra S, Beer RH, Brenowitz M. 2006. Fast Fenton footprinting: A laboratory-based method for the time-resolved analysis of DNA, RNA and proteins. Nucleic Acids Res 34: e48. doi: 10.1093/nar/gkl055.

Showalter SA, Hall KB. 2005. Isotropic reorientational eigenmode dynamics complements NMR relaxation measurements for RNA. Methods Enzymol 394: 465-480.

Stone, M.D., Mihalusova, M., O'Connor C, M., Prathapam, R., Collins, K., and Zhuang, X. 2007. Stepwise protein-mediated RNA folding directs assembly of telomerase ribonucleoprotein. Nature 446: 458-461.

Sun X, Zhang Q, Al-Hashimi HM. 2007. Resolving fast and slow motions in the internal loop containing stem-loop 1 of HIV-1 that are modulated by $\mathrm{Mg}^{2+}$ binding: Role in the kissing-duplex structural transition. Nucleic Acids Res 35: 1698-1713.

Tjandra N, Bax A. 1997. Direct measurement of distances and angles in biomolecules by NMR in a dilute liquid crystalline medium. Science 278: 1111-1114.

Tolman JR. 2002. A novel approach to the retrieval of structural and dynamic information from residual dipolar couplings using several oriented media in biomolecular NMR spectroscopy. J Am Chem Soc 124: 12020-12030.

Tolman JR, Flanagan JM, Kennedy MA, Prestegard JH. 1995. Nuclear magnetic dipole interactions in field-oriented proteins: Informa- tion for structure determination in solution. Proc Natl Acad Sci 92: 9279-9283.

Tolman JR, Flanagan JM, Kennedy MA, Prestegard JH. 1997. NMR evidence for slow collective motions in cyanometmyoglobin. Nat Struct Biol 4: 292-297.

Vicens Q, Westhof E. 2003. Molecular recognition of aminoglycoside antibiotics by ribosomal RNA and resistance enzymes: An analysis of x-ray crystal structures. Biopolymers 70: 42-57.

Weeks KM, Cech TR. 1995. Protein facilitation of group I intron splicing by assembly of the catalytic core and the $5^{\prime}$ splice site domain. Cell 82: 221-230.

Weeks KM, Cech TR. 1996. Assembly of a ribonucleoprotein catalyst by tertiary structure capture. Science 271: $345-348$.

Williamson JR. 2000. Induced fit in RNA-protein recognition. Nat Struct Biol 7: 834-837.

Williamson JR. 2008. Biophysical studies of bacterial ribosome assembly. Curr Opin Struct Biol 18: 299-304.

Woodson SA. 2008. RNA folding and ribosome assembly. Curr Opin Chem Biol 12: 667-673.

Xia T. 2008. Taking femtosecond snapshots of RNA conformational dynamics and complexity. Curr Opin Chem Biol 12: 604-611.

Zhang Q, Throolin R, Pitt SW, Serganov A, Al-Hashimi HM. 2003. Probing motions between equivalent RNA domains using magnetic field induced residual dipolar couplings: Accounting for correlations between motions and alignment. J Am Chem Soc 125: 10530-10531.

Zhang Q, Sun X, Watt ED, Al-Hashimi HM. 2006. Resolving the motional modes that code for RNA adaptation. Science 311: 653656.

Zhang Q, Stelzer AC, Fisher CK, Al-Hashimi HM. 2007. Visualizing spatially correlated dynamics that directs RNA conformational transitions. Nature 450: 1263-1267. 

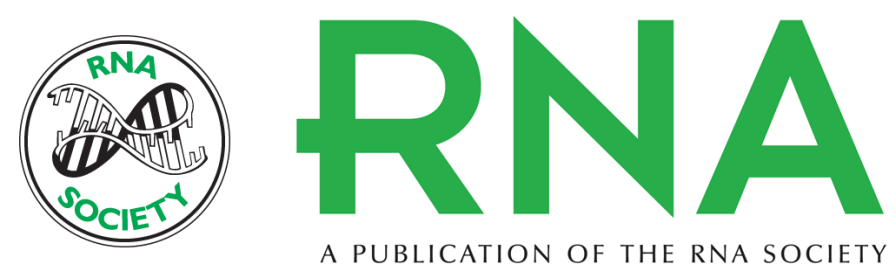

A PUBLICATION OF THE RNA SOCIETY

\section{Domain-elongation NMR spectroscopy yields new insights into RNA dynamics and adaptive recognition}

Qi Zhang and Hashim M. Al-Hashimi

RNA 2009 15: 1941-1948 originally published online September 23, 2009

Access the most recent version at doi:10.1261/rna.1806909

$\begin{array}{ll}\text { References } & \begin{array}{l}\text { This article cites } 72 \text { articles, } 16 \text { of which can be accessed free at: } \\ \text { http://rnajournal.cshlp.org/content/15/11/1941.full.html\#ref-list-1 }\end{array}\end{array}$

License

Email Alerting Receive free email alerts when new articles cite this article - sign up in the box at the Service top right corner of the article or click here.

To subscribe to $R N A$ go to:

http://rnajournal.cshlp.org/subscriptions 